\title{
Acetylcholine receptor $\delta$ subunit mutations underlie a fast-channel myasthenic syndrome and arthrogryposis multiplex congenita
}

\author{
Sharon Brownlow, ${ }^{1}$ Richard Webster, ${ }^{1}$ Rebecca Croxen, ${ }^{1}$ Martin Brydson, ${ }^{1}$ Brian Neville, ${ }^{2}$ \\ Jean-Pierre Lin, ${ }^{3}$ Angela Vincent, ${ }^{1}$ John Newsom-Davis, ${ }^{1}$ and David Beeson ${ }^{1}$
}

${ }^{1}$ Neurosciences Group, Weatherall Institute of Molecular Medicine, John Radcliffe Hospital, Headington, Oxford, United Kingdom

${ }^{2}$ Great Ormond Street Hospital, London, United Kingdom

${ }^{3}$ Guy's Hospital, London, United Kingdom

Address correspondence to: David Beeson, Neurosciences Group, Weatherall Institute of Molecular Medicine, John Radcliffe Hospital, Headington, Oxford OX3 9DS, United Kingdom.

Phone: 44-0-1865-222311; Fax: 44-0-1865-222402; E-mail: dbeeson@hammer.imm.ox.ac.uk.

Received for publication April 5, 2001, and accepted in revised form May 21, 2001.

Limitation of movement during fetal development may lead to multiple joint contractures in the neonate, termed arthrogryposis multiplex congenita. Neuromuscular disorders are among the many different causes of reduced fetal movement. Many congenital myasthenic syndromes (CMSs) are due to mutations of the adult-specific $\varepsilon$ subunit of the acetylcholine receptor (AChR), and, thus, functional deficits do not arise until late in gestation. However, an earlier effect on the fetus might be predicted with some defects of other AChR subunits. We studied a child who presented at birth with joint contractures and was subsequently found to have a CMS. Mutational screening revealed heteroallelic mutation within the AChR $\delta$ subunit gene, $\delta 756$ ins 2 and $\delta \mathrm{E} 59 \mathrm{~K}$. Expression studies demonstrate that $\delta 756 \mathrm{ins} 2$ is a null mutation. By contrast, both fetal and adult AChR containing $\delta \mathrm{E} 59 \mathrm{~K}$ have shorter than normal channel activations that predict fast decay of endplate currents. Thus, $\delta \mathrm{E} 59 \mathrm{~K}$ causes dysfunction of fetal as well as the adult $\mathrm{AChR}$ and would explain the presence of joint contractures on the basis of reduced fetal movement. This is the first report of the association of AChR gene mutations with arthrogryposis multiplex congenita. It is probable that mutations that severely disrupt function of fetal AChR will underlie additional cases.

J. Clin. Invest. 108:125-130 (2001). DOI:10.1172/JCI200112935.

\section{Introduction}

Arthrogryposis multiplex congenita (AMC) is a term used to describe a heterogeneous group of disorders characterized by nonprogressive joint contractures that are present at birth (1). The causes are diverse, as any process that limits fetal movements during development may lead to congenital joint contractures. Moreover, because these conditions differ in their course and prognosis, require different treatments, and may have genetic implications, it is important to ascertain the underlying cause. Among causes that have been identified are disorders of neuromuscular transmission. On rare occasions, AMC may associate with myasthenia gravis, an autoimmune disorder in which autoantibodies target the muscle acetylcholine receptors (AChRs) that are present on the postsynaptic membrane at the neuromuscular junction. AChRs exist in two forms: one that is expressed during fetal development (fetal AChR) consisting of $\alpha_{2} \beta \gamma \delta$ subunits, and the other, in which an $\varepsilon$ subunit replaces the $\gamma$, that is present at the motor endplates in mature muscle (adult AChR, $\alpha_{2} \beta \varepsilon \delta$ ) (2). In a number of cases, it has been shown that AMC is due to autoantibodies that specifically inhibit function of fetal but not adult $\operatorname{AChR}(3,4)$. Rarely, AMC has also been reported in association with hereditary/congenital myasthenic syndromes (CMSs), but the underlying genetic cause has not been elucidated $(5,6)$.

The phenotype of the fast-channel syndrome is similar to the more common AChR-deficiency syndrome. Patients present in the neonatal period with ocular, bulbar, or respiratory symptoms; excessive fatigability; and delayed motor milestones. Both disorders show recessive inheritance. Fast-channel syndromes have been defined by molecular and electrophysiological studies. These show that certain mutations within the AChR subunit genes affect AChR kinetics, causing infrequent and brief ion channel activations (7-9). Phenotypic effects of these mutations are seen when the fast-channel allele is inherited recessively or in combination with a low expressor or null allele. This unmasks the effects of the fast-channel mutation and generates postsynaptic adult-type AChRs that have a reduced response to ACh.

To our knowledge, our report here is the first full description of mutations in the AChR $\delta$ subunit gene, which were identified in a patient with AMC and a CMS. We identify a missense mutation in the extracellular domain that shortens channel activations of both 
adult and fetal AChR subtypes. The phenotypic effects of this mutation are unmasked by the presence of a null mutation on the second allele. Dysfunction of the fetal AChR probably underlies the AMC, and dysfunction of the adult AChR is likely to cause the fast-channel CMS phenotype.

\section{Methods}

DNA samples. Genomic DNA was isolated from peripheral blood using the Nucleon II DNA extraction kit (Nucleon Biosciences, Glasgow, United Kingdom). Approval for the use of human tissues was received from the Central Oxford Research Ethics Committee. Mutational analysis. PCR and single-strand conformational polymorphism (SSCP) analysis were performed as described previously (10). Intronic sequences within the AChR subunit genes were obtained by analyzing genomic clones containing each of the AChR subunit genes (11). Oligonucleotide primers were designed for amplification of each of the exons and their respective intron/exon boundaries. A typical PCR reaction included reaction buffer $[60 \mathrm{mM}$ Tris-HCl, $15 \mathrm{mM}\left(\mathrm{NH}_{4}\right)_{2} \mathrm{SO}_{4}, 1.5-2.0 \mathrm{mM} \mathrm{MgCl}_{2}(\mathrm{pH}$ 8.5); Invitrogen Corp., San Diego, California, USA], $1.25 \mu \mathrm{M}$ each primer, $200 \mu \mathrm{M}$ dCTP, dGTP, and dTTP, $0.075 \mu \mathrm{l}$ of $\left.{ }^{35} \mathrm{~S}\right] \mathrm{dATP} \alpha \mathrm{S}(>1,000 \mathrm{Ci} / \mathrm{mmol}$; Amersham Pharmacia Biotech, Piscataway, New Jersey, USA), 50 $\mu \mathrm{M}$ dATP with about $50 \mathrm{ng}$ of genomic DNA, and 0.25 U Taq DNA polymerase (PE Biosystems, Warrington, Cheshire, United Kingdom) in a 5- $\mu$ l reaction. The resulting PCR amplicons were analyzed by SSCP. Primer pairs $5^{\prime}$-TATGGCCCTACCGTCTAATTAC- $3^{\prime}$ and $5^{\prime}$-TCGGCCTTCCAAAGTGCTGG- ${ }^{\prime}$, and $5^{\prime}$-CTAAGATGTCCATGTGCCGC- $3^{\prime}$ and $5^{\prime}$-GGACGGGCCTGGAGGCTC- $3^{\prime}$ were used for amplification of AChR $\delta$ subunit gene exons 3 and 7 respectively. Amplicons that showed abnormal conformers were purified from agarose gels by using the QIAGEN QIAquick gel extraction kit (QIAGEN Inc., Valencia, California, USA) and were subjected to direct DNA sequencing (DNA sequencing facility, Department of Biochemistry, Oxford University, United Kingdom). DNA sequence changes were confirmed by PCR, and restriction endonuclease digestions were carried out according to the manufacturer's instructions.

Expression constructs. cDNAs encoding the $\mathrm{AChR} \alpha, \beta$, $\delta$, $\varepsilon$, and $\gamma$ subunits (12) were subcloned into pcDNA3.1 (Invitrogen Corp.). Mutagenic primers 5'-CAATGT-

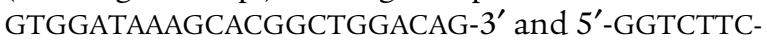
TACCTACCGGCTGACTGGTGAGAAGACATCAGTGGCC-3' were used to introduce the $\delta \mathrm{E} 59 \mathrm{~K}$ and $\delta 756 \mathrm{del} 2 \mathrm{muta}$ tions into the wild-type AChR $\delta$ subunit sequence using the GeneEditor in vitro site-directed mutagenesis system (Promega Corp., Madison, Wisconsin, USA). The resultant $\delta$ subunit cDNAs were sequenced to check for the presence of the mutations and absence of any additional sequence changes.

Expression studies. Respective mutant $\delta$ subunit cDNAs, in combination with wild-type $\alpha, \beta, \varepsilon$, or $\gamma$ were transfected into HEK293 cells. The cells were grown on six-well tissue culture plates and transfected using polyethylenimine. Surface AChR expression was measured by overlaying the cells in PBS containing $10 \mathrm{nM}{ }^{125} \mathrm{I}-\alpha-$ bungarotoxin ( $\left.{ }^{125} \mathrm{I}-\alpha-\mathrm{BuTx}\right)$ and $1 \mathrm{mg} / \mathrm{ml}$ BSA for 1 hour. Cells were washed four times with PBS and removed from the plate in $60 \mathrm{mM}$ Tris- $\mathrm{HCl}(\mathrm{pH} 7.4)$, $150 \mathrm{mM} \mathrm{NaCl}, 5 \mathrm{mM}$ EDTA, $0.5 \mathrm{mM}$ phenylmethylsulphonyl fluoride, and $1.25 \%$ Triton X-100, and the amount of bound ${ }^{125} \mathrm{I}-\alpha$-BuTx was determined. Binding of ${ }^{125} \mathrm{I}-\alpha$-BuTx to $\delta$ subunit-containing surface AChR was established by immunoprecipitation with the $\delta$ subunit-specific mAb C7 (13). Immunoprecipitation with an $\alpha$ subunit-specific polyclonal serum was used to determine total cell ${ }^{125} \mathrm{I}-\alpha$-BuTx binding in Triton X-100 extracts of HEK293 transfected with $\alpha \delta$ cDNAs (14). Levels of ${ }^{125} \mathrm{I}-\alpha$-BuTx binding in extracts of $\alpha \delta$ and $\alpha \delta \mathrm{E} 59 \mathrm{~K}$-transfected cells were determined, and then equal quantities were used in immunoprecipitation using increasing ${ }^{125} \mathrm{I}-\alpha$-BuTx concentrations.

Patch clamp recordings and analysis. Recordings were performed in the cell-attached patch configuration (15) at $20-22^{\circ} \mathrm{C}$. Cells were bathed in a solution containing $150 \mathrm{mM} \mathrm{NaCl}, 2.8 \mathrm{mM} \mathrm{KCl}, 2 \mathrm{mM} \mathrm{MgCl}_{2}, 1$ $\mathrm{mM} \mathrm{CaCl}, \quad 10 \mathrm{mM}$ glucose; and $10 \mathrm{mM}$ HEPES/NaOH ( $\mathrm{pH}$ 7.4). The pipette solution contained the above buffer without glucose but with ACh. Single-channel currents were amplified with an Axopatch 200B amplifier (Axon Instruments Inc., Foster City, California, USA), sampled to hard disk at 90 $\mathrm{kHz}$, initially filtered at $9 \mathrm{kHz}(-3 \mathrm{~dB}$, Bessel filter). Data were further filtered for analysis to a final cumulative corner frequency $\left(f_{c}\right)$ of $4.37 \mathrm{kHz}$ with resolution set at 45 microseconds. Recordings were made with a pipette potential set at $+80 \mathrm{mV}$. Channel transitions were detected by $50 \%$ amplitude threshold crossings (pClamp6). Current amplitude was estimated by construction of all-point histograms from well-defined full openings, fitted by maximum log likelihood of two Gaussian functions. Bursts were defined as groups of openings separated by closed intervals longer than a critical duration $\left(t_{\text {crit }}\right)$; $t_{\text {crit }}$ was chosen so that equal numbers of short and long intervals are misclassified (16). Histograms of burst duration were fitted to the sum of exponentials by maximum log likelihood. At concentrations between 10 and $500 \mu \mathrm{M}$, AChR activity occurred in clusters of individual channel openings, thus enabling estimation of the channel open probability at different ACh concentrations. Clusters were defined as groups of openings separated by a period 10 times longer than the predominant (interburst) closed interval population for each patch studied (about 0.1-10 milliseconds). Only clusters longer than 100 milliseconds were considered for estimation of open probability. At least 20 clusters from each patch were analyzed. Values are mean \pm SEM, and significant differences between groups were determined by unpaired Student's $t$ test, with $P<0.05$ defined as a significant difference. 
a

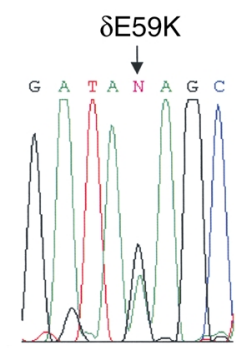

Mutant TGG ATA AAG CAC Trp Ile Lys His

Wild-type TGG ATA GAG CAC Trp Ile Glu His

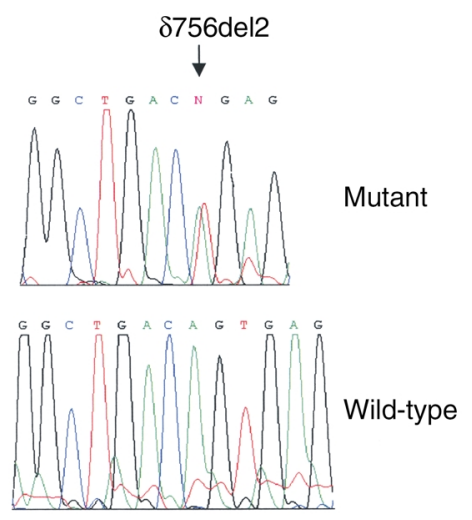

C

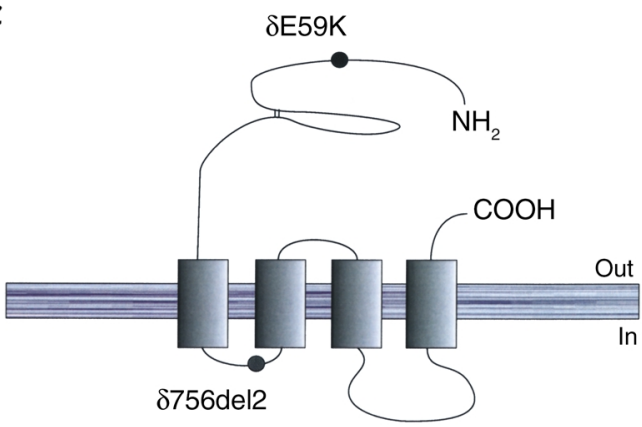

d $\delta$ subunits

b
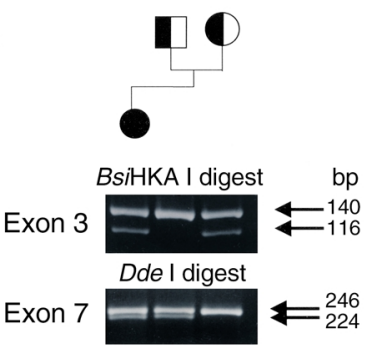

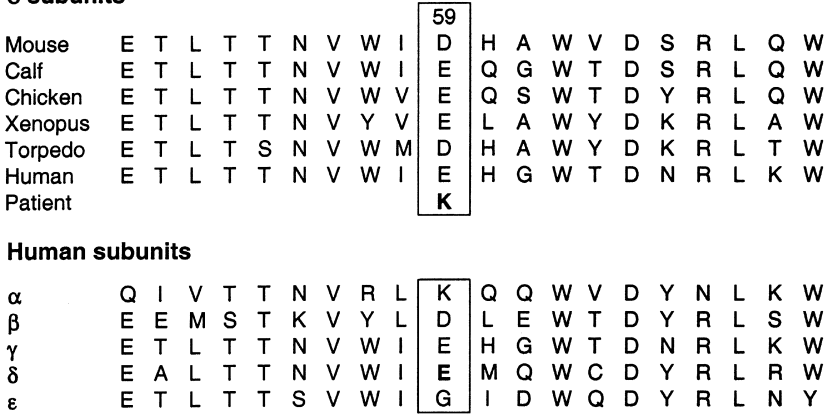

Figure 1

(a) DNA sequence analysis of PCR amplicons from patient and control DNA samples showing a G $\rightarrow$ A transition in exon 3 and an AG dinucleotide deletion in exon 7; both are heterozygous. (b) Restriction endonuclease digestion analysis of PCR amplicons from propositus and parents. Digests were subjected to electrophoresis on $2 \%$ agarose/TBE gels and visualized under ultraviolet light. A BsiHKA I digest of the exon 3 amplicon shows $\delta \mathrm{E} 59 \mathrm{~K}$ is inherited from the mother, and a Ddel digest of the exon 7 amplicon shows $\delta 756$ del 2 is inherited from the father. (c) Schematic representation of the position of the mutations within the $\delta$ subunit. (d) Multiple alignments of deduced amino acid sequences in the vicinity of the $\delta \mathrm{E} 59 \mathrm{~K}$ mutation in the $\mathrm{N}$-terminal extracellular domain for the human AChR subunits and for $\delta$ subunits from six species.

\section{Results}

Clinical features. The propositus, now 6 years old, is the fourth child of unrelated parents who have no neurological disorder. Fetal movements were thought to be reduced compared with those of her surviving sibs. She had fixed flexion contractures of the interphalangeal joints of both hands. She choked while feeding and developed hypoxia on the day of birth. She was noted to be floppy with a weak cry and had feeding difficulties. Over the next few months, she had several episodes of severe respiratory difficulty and cyanosis with secondary seizures, and assisted ventilation was required for a brief period.

At the age of 4 months, she was admitted to Great Ormond Street Children's Hospital. An edrophonium test was positive, and single-fiber electromyography showed increased jitter with blocking. Anti-AChR antibodies were not detected. A diagnosis of congenital myasthenia was made (J.-P. Lin), and treatment with pyridostigmine led to improvement in strength.

She walked at 14 months. At the age of 6 years, while taking regular pyridostigmine treatment, she had fatigable ptosis, limitation of eye movements, and intermittent swallowing difficulty. There was fatigable weakness of upper and lower limbs. She was unable to walk more than 400 meters, and would tire when climbing stairs.
Two older brothers are well, but a third brother had reduced fetal movements and presented at birth with similar symptoms to those of the propositus, including "crablike" hands and bilateral ptosis. He died with bronchopneumonia at the age of 5 months.

Mutational analysis. Screening of promoter regions and all exons within the genes encoding the AChR $\alpha$, $\beta, \delta$, and $\varepsilon$ subunits by SSCP analysis revealed two abnormal conformers, one in exon 3 and one in exon 7 of the AChR $\delta$ subunit gene. Direct sequencing of the exon 3 amplicon showed a $G \rightarrow A$ transition at nucleotide position 175 , which results in the missense amino acid substitution, $\delta \mathrm{E} 59 \mathrm{~K}$. Direct sequencing of the exon 7 amplicon showed a dinucleotide AG deletion, $8756 \mathrm{del} 2$, at the exon/intron boundary (Figure 1a). Restriction endonuclease digestions with $B s i \mathrm{HKA}$ I and $D d e I$ were used to confirm the mutations and to demonstrate that $\delta \mathrm{E} 59 \mathrm{~K}$ is inherited from the mother and $\delta 756 \mathrm{del} 2$ from the father (Figure $1 \mathrm{~b}$ ). $\delta \mathrm{E} 59$ is in an extracellular region that is well conserved between species, and each species has a negatively charged residue (either Glu or Asp) at position $\delta 59$ (Figure 1, c and $d$ ). However, the region is less well conserved between subunits (Figure 1d). $\delta 756 \mathrm{del} 2$ is at the $\delta$-gene exon 7 /intron 7 boundary and is likely to result in the loss of exon 7 from the $\delta$ subunit mRNA transcript. Other possibilities are normal excision of intron 7 , 
Figure 2

(a) Expression of adult- and fetal-type AChR containing mutant $\delta$ E59K and $\delta 756$ del 2 subunits in HEK293 cells. ${ }^{125} \mathrm{I}-\alpha$-BuTx binding to surface AChRs containing the mutant $\delta$ subunits expressed in HEK293 cells. Cell-surface AChR (total, left) and cell-surface AChR precipitated by an anti- $\delta$-subunit-specific antibody (anti- $\delta$, right) from transfected HEK293 cells. Results are normalized for ${ }^{125} \mathrm{I}-\alpha-\mathrm{B} u T \mathrm{~T}$ binding to $\alpha \beta \delta \varepsilon$ and represent the mean \pm SD of four experiments. (b and c) Triton X-100 extracts of HEK 293 cells transfected with $\alpha, \alpha \delta$, and $\alpha \delta$-mutant cDNAs. (b) ${ }^{125}$ I- $\alpha$-BuTx binding to $\alpha, \alpha \delta, \alpha \delta \mathrm{E} 59 \mathrm{~K}$, and $\alpha \delta 756 \mathrm{del} 2$. (c) Dose-response relationship of ${ }^{125} \mathrm{I}-\alpha$-BuTx binding to $\alpha \delta$ and $\alpha \delta$ E59K.

which would lead to a frameshift, or retention of intron 7, which would result in an immediate stop codon. A muscle biopsy from the patient was not available to study splicing of the $\delta$ subunit RNA transcripts, but given that each of these possibilities will result in a nonfunctional $\delta$ subunit, $\delta 756 \mathrm{del} 2$ is almost certainly a null mutation.

Expression studies. Because the patient phenotype suggested an effect of the mutations in utero as well as on mature muscle, we investigated functional effects of the $\delta$ subunit mutations on both fetal- and adult-type human AChR. cDNAs encoding mutant $\delta$ subunits in combination with wild-type $\alpha, \beta, \varepsilon$, or $\gamma$ were transfected into HEK293 cells, and cell surface ${ }^{125} \mathrm{I}-\alpha$-BuTx was measured. To prove that surface ${ }^{125} \mathrm{I}-\alpha-\mathrm{BuT}$ - $\mathrm{x}$ binding was due to AChR containing $\delta$ subunits, we immunoprecipitated with a $\delta$ subunit-specific $m A b$ (Figure 2a). $\alpha \beta \delta \mathrm{E} 59 \mathrm{~K} \varepsilon$ gave robust surface ${ }^{125} \mathrm{I}-\alpha-\mathrm{BuTx}$ binding, indicating surface expression of AChR pentamers containing this mutation, whereas $\alpha \beta \delta 756 \mathrm{del} 2 \varepsilon$ gave little or no surface binding. Similarly, wild-type $\alpha \beta \gamma$ and $\alpha \beta \varepsilon$ gave little or no surface binding. For both adult- and fetal-type AChR containing $\delta$ E59K, surface binding was reduced to around $60 \%$ of the levels seen for the respective wild-type receptors. Similarly, reduced ${ }^{125}$ I$\alpha$-BuTx binding was seen in Triton X-100 extracts of HEK293 cells transfected with $\alpha \delta$ and $\alpha \delta E 59 K$ (Figure $2 b)$. This appears to reflect reduced expression/assembly of the $\alpha \delta \mathrm{E} 59 \mathrm{~K}$ dimers, rather than any change in affinity for ${ }^{125} \mathrm{I}-\alpha$-BuTx, as wild-type $\alpha \delta$ and $\alpha \delta \mathrm{E} 59 \mathrm{~K}$ dimers (or full AChRs) showed similar ${ }^{125} \mathrm{I}-\alpha-\mathrm{BuTx}$ binding curves (Figure 2c).

Electrophysiology. To confirm pathogenicity of $\delta \mathrm{E} 59 \mathrm{~K}$, we compared single-channel currents recorded from a

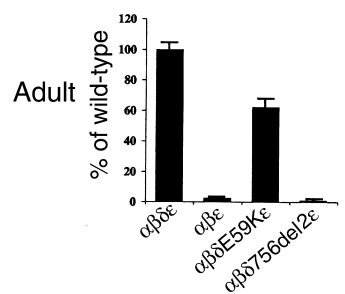

Surface AChR precipitated by anti- $\delta$
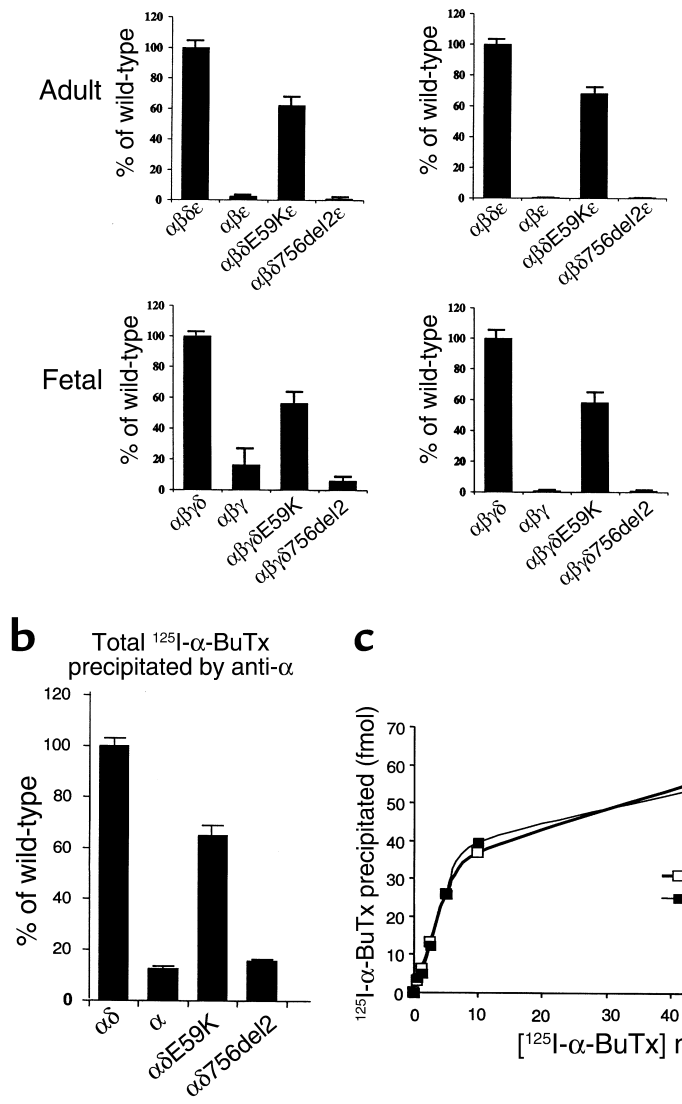

C

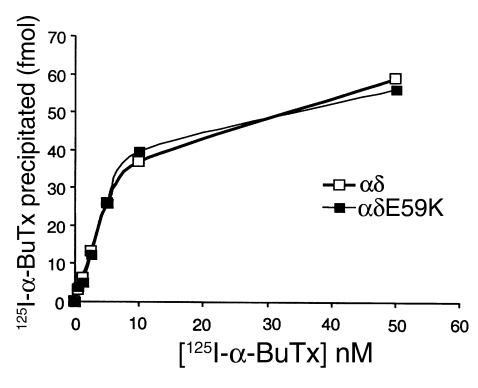

HEK293 cells transfected with either wild-type or mutant AChR cDNAs. First, we investigated the open probability of the adult wild-type and mutant AChRs at relatively high concentrations of ACh. At concentrations between 10 and $500 \mu \mathrm{M}, \mathrm{AChR}$ activity occurred in clusters of openings from individual channels separated by longer closed periods, and thus enabled estimation of channel open probability at the different ACh concentrations. Example recordings of wild-type and mutant adult AChR cluster activity at $30 \mu \mathrm{M}$ ACh (Figure 3a) demonstrate the open probability of the AChR within a cluster is high for wild-type $(0.69 \pm 0.01)$ but low for the mutant $(0.175 \pm 0.02)$. Thus, within a cluster, the mutant channels spend a far shorter period in the open state. The ACh dose-response curves of open probability give an $\mathrm{EC}_{50}$ of 14.2 and $58.4 \mu \mathrm{M} \mathrm{ACh}$

\section{Figure 3}

Acetylcholine dose-response relationship of adult wild-type and adult $\delta$ E59K-AChR expressed in HEK293 cells. (a) Cluster activity of adult AChR or adult $\delta$ E59K-AChR in the presence of $30 \mu \mathrm{M}$ ACh. (b) The dose-response relationship between channel open probability within a defined cluster and the concentration of ACh (open circles, adult wild-type; filled circles, adult $\delta$ E59K-AChR). Each data point represents results from one patch. Data were fitted to the logistic function. The $\mathrm{EC}_{50}$ was 14.2 and $58.4 \mu \mathrm{M}$ for wild-type and adult SE59K-AChR, respectively.
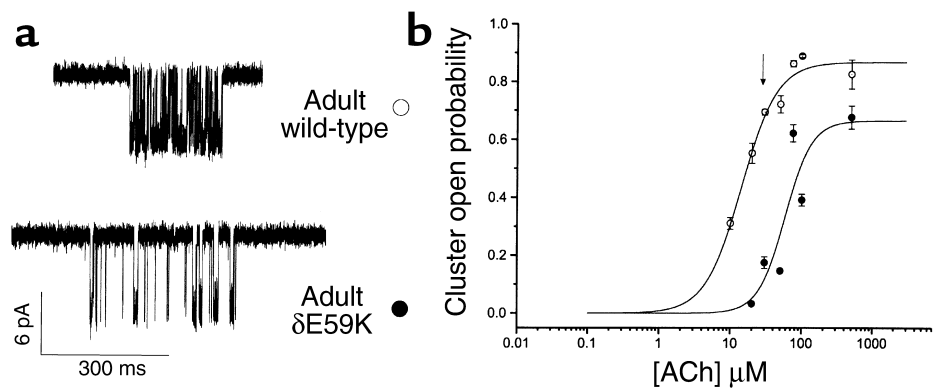
Figure 4

Single-channel currents of human adult and fetal AChR and adult $\delta$ E59K-AChR and fetal $\delta$ E59K-AChR expressed in HEK293 cells. The left panel shows examples of single-channel activations (openings are downward deflections, filtered at $4.37 \mathrm{kHz}$ ). Scale bars refer to all example traces. The right panel shows the respective burst duration histograms fitted by the sum of three exponential functions; the longest burst-length component is indicated for each histogram.

for wild-type adult and mutant adult AChR, respectively (Figure $3 b$ ).

We next looked at burst duration of both adult and fetal wild-type AChR compared with adult and fetal AChR containing SE59K (Figure 4 and Table 1). Wild-type and mutant AChR burst-duration distributions could be fitted by three burst-length components. The third (or longest) burst length component, $t_{3}$, is the major determinant of ion channel characteristics. AChR containing $\delta \mathrm{E} 59 \mathrm{~K}$ caused a significant reduction in burst duration of this longest component for both adult and fetal receptors; for adult AChR, $t_{3}(\mathrm{~ms}) 5.06 \pm 0.46 \rightarrow 2.75 \pm 0.05$ ( $\left.P<0.01\right)$; for fetal AChR, $t_{3}(\mathrm{~ms}) 10.95 \pm 1.13 \rightarrow 6.48 \pm 0.05(P<0.03)$. Thus both adult- and fetal-type AChRs containing the $\delta \mathrm{E} 59 \mathrm{~K}$ mutation are likely to have both fewer and shorter channel activations.

\section{Discussion}

To our knowledge, this is the first full report of mutations within the AChR $\delta$ subunit gene underlying CMS. This CMS associates with arthrogryposis multiplex congenita, and we provide evidence that the $\delta$ subunit mutations affect both adult and fetal AChR subtypes and thus are the probable cause of both disorders. Inheritance of $\delta 756 \mathrm{del} 2$, a null mutation, unmasks the effects of a second mutation, $\delta \mathrm{E} 59 \mathrm{~K}$. Expression studies using HEK293 cells show that mutant AChR containing $\delta \mathrm{E} 59 \mathrm{~K}$ have "fast-channel syndrome" characteristics of abnormally brief channel activation episodes. This mutation also appears to cause a modest reduction in AChR surface expression (although levels of expression in HEK293 cells do not always correlate precisely with in vivo observations). It is likely that a combination of both of these properties is responsible for reduced responses to $\mathrm{ACh}$ of adult AChRs at fully mature endplates and of fetal AChRs at developing endplates and, thus, would explain the patient phenotype.

The propositus has a fast-channel syndrome. Anecdotally we have noted that fast-channel syndrome patients, in particular,

Table 1

Burst durations
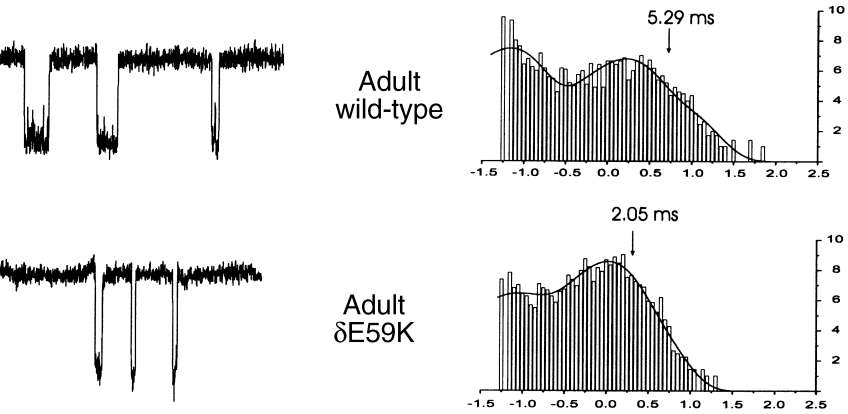

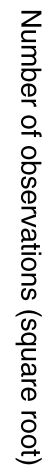

Fetal
wild-type
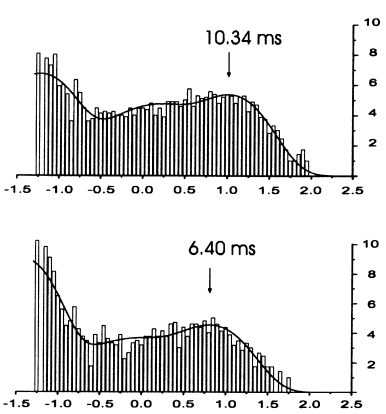

Burst duration (log ms)

may suffer episodes of severe apnea in childhood that may be life threatening (J. Newsom-Davis and D. Beeson, unpublished observations). Indeed, the patient's brother, who showed similar symptoms, died at the age of 5 months. Life-threatening respiratory problems are also commonly seen in CMS-EA, a congenital myasthenic syndrome caused by mutations in the presynaptic protein choline acetyltransferase (17). Thus, for prognosis and management, diagnostic precision for CMS may be crucial.

Fast-channel kinetics have been reported for four other mutations that underlie CMS, عL121P (7), عR311W (18), $\varepsilon 1254$ ins 18 (8), and $\alpha$ V285I (9). The $\varepsilon R 311 \mathrm{~W}$, $\varepsilon 1254$ ins 18 , and $\alpha$ V285I mutations were associated with severe reduction of $\alpha$-BuTx binding sites at endplates in patient muscle biopsies, whereas, for two unrelated patients with EL121P, the density and distribution of AChR on the junctional folds were normal. Detailed kinetic analysis of $\varepsilon$ L121P shows that it results in diminished agonist binding affinity, reduced channel reopenings during ACh occupancy, and decreased desensitization of the mutant AChR by ACh (7). The primary effect

\begin{tabular}{|c|c|c|c|c|c|}
\hline Receptor type & Amplitude (pA) & $t_{1}(\mathrm{~ms})$ & $t_{2}(\mathrm{~ms})$ & $t_{3}(\mathrm{~ms})$ & $\begin{array}{l}\text { Number } \\
\text { of patches }\end{array}$ \\
\hline Adul & 29 & 0.067 & 1.28 & 5.0 & 5 \\
\hline$d u$ & 9.1 & 0. & 2 & $2.75 \pm$ & 5 \\
\hline Fetal wild-type & $6.03 \pm 0.17$ & $0.051 \pm 0.003$ & $0.76 \pm 0.15$ & $10.95 \pm 1.13$ & 6 \\
\hline Fetal $\delta \mathrm{E} 59 \mathrm{~K}$ & $5.56 \pm 0.34$ & $0.045 \pm 0.003$ & $0.75 \pm 0.25$ & $6.48 \pm 1.32^{\mathrm{A}}$ & 7 \\
\hline
\end{tabular}

Single-channel current amplitude $(\mathrm{pA})$ and burst-length time constants, $t_{\mathrm{n}}$, are presented as mean $\pm \mathrm{SE}$ The longest component $t_{3}$ is shown in bold. ASignificant difference (see the text) between equivalent characteristics of wild-type and mutant receptor. 
of the $\delta \mathrm{E} 59 \mathrm{~K}$ mutation may also be on agonist binding affinity. $\delta \mathrm{E} 59 \mathrm{~K}$ is in a region of the $\delta$ subunit that is thought to contribute to agonist binding. In photoaffinity labeling studies on Torpedo, AChR homologous residues $\gamma$-Trp55 and $\delta$-Trp57 incorporated $\left[{ }^{3} \mathrm{H}\right] d$ tubocurarine $(19,20)$, and in vitro mutagenesis studies on mouse AChR showed the missense mutation $\delta \mathrm{D} 59 \mathrm{E}$ altered affinity for carbamylcholine when the receptor is in the resting state (21). In $\delta \mathrm{E} 59 \mathrm{~K}$, a negatively charged glutamate is replaced by a positively charged lysine residue. Because ACh contains a positively charged quarternary ammonium group, $\delta \mathrm{E} 59 \mathrm{~K}$ would be expected to affect ACh binding affinity. However, detailed kinetic analysis of this mutation will be required to establish precisely how it affects AChR function. $\delta \mathrm{E} 59 \mathrm{~K}$ did not affect $\alpha$-BuTx binding. This result is consistent with studies of the homologous region of $\alpha 7$ neuronal nicotinic AChR in which mutation of $\alpha 7-\operatorname{Trp} 54$ caused a decrease in binding affinity for ACh without significant modification of $\alpha$-BuTx binding (22).

CMS are not uncommon, and at least 70 different mutations have been identified (23-26). However, documentation of the association with AMC is rare. The majority of CMSs are due to mutations within the AChR $\varepsilon$ subunit gene. In humans, the $\varepsilon$ subunit is only fully expressed late in gestation and therefore mutations of the $\varepsilon$ subunit would not be expected to affect fetal development. Indeed AMC has not been noted in patients with either fast-channel or AChR deficiency syndromes due to $\varepsilon$ subunit gene mutations. Similarly, although mutations that underlie the slow channel myasthenic syndrome are located in each of the AChR subunits $(23,24)$, they result in an increased response to $\mathrm{ACh}$ and would not inhibit fetal movement. By contrast, mutations in subunits of the fetal receptor that result in a reduced response to ACh might be expected to inhibit seriously fetal movement during crucial developmental periods. However, features of AMC were not noted in a case of AChR deficiency due to a 3-codon deletion of the $\beta$ subunit that affects AChR assembly (27) or in a case of fast-channel syndrome due to a missense mutation in the $\alpha$ subunit M3 region (9). It may be that AMC was present in these cases but not documented or that these mutations did not cause sufficient dysfunction of fetal AChR to result in AMC. Studies of autoimmune myasthenia clearly show that maternal antibodies that inhibit fetal AChR can associate with $\operatorname{AMC}(3,4)$. Here we demonstrate that the AChR $\delta \mathrm{E} 59 \mathrm{~K}$ mutation also affects fetal AChR function. It is therefore highly likely that the $\delta \mathrm{E} 59 \mathrm{~K}$ mutation underlies both a fast-channel syndrome and the associated AMC. Our results suggest the genes encoding the $\operatorname{AChR} \alpha, \beta$, and $\delta$ subunits, as well as choline acetyltransferase, should be primary candidates when screening for mutations that underlie cases of AMC associated with a CMS.

\section{Acknowledgments}

This work was supported by the MRC and the Muscular Dystrophy Campaign/Myasthenia Gravis Association.
1. Hall, J.H. 1996. Arthrogryposis (multiple congenital contractures). In Principles and practice of medical genetics. 3 rd edition. A.E. Emery and D.L. Rimoin, editors. Churchill Livingstone. New York, New York, USA. 2869-2915

2. Mishina, M., et al. 1986. Molecular distinction between fetal and adult forms of muscle acetylcholine receptor. Nature. 321:406-411.

3. Vincent, A., et al. 1995. Arthrogryposis multiplex congenita with maternal antibodies specific for fetal antigen. Lancet. 346:24-25.

4. Riemersma, S., et al. 1996. Association of arthrogryposis multiplex congenita with maternal antibodies inhibiting fetal acetylcholine receptor function. J. Clin. Invest. 98:2358-2363.

5. Smit, L.M., and Barth, P.G. 1980. Arthrogryposis multiplex congenita due to congenital myasthenia. Dev. Med. Child Neurol. 22:371-374.

6. Vajsar, J., et al. 1995. Arthrogryposis multiplex congenita due to congenital myasthenic syndrome. Pediatr. Neurol. 12:237-241.

7. Ohno, K., et al. 1996. Congenital myasthenic syndrome caused by decreased agonist binding affinity due to a mutation in the acetylcholine receptor $\varepsilon$ subunit. Neuron. 17:157-170.

8. Milone, M., et al. 1998. Mode switching kinetics produced by a naturally occurring mutation in the cytoplasmic loop of the human acetylcholine receptor $\varepsilon$ subunit. Neuron. 20:575-588.

9. Wang, H.-L., et al. 1999. Acetylcholine receptor M3 domain: stereochemical and volume contributions to channel gating. Nat. Neurosci. 2:226-233.

10. Croxen, R., et al. 1997. Mutations in different functional domains of the human muscle acetylcholine receptor $\alpha$ subunit in patients with slow-channel congenital myasthenic syndrome. Hum. Mol. Genet. 6:767-774.

11. Beeson, D., et al. 1993. cDNA and genomic clones encoding the human muscle acetylcholine receptor. Ann. NY Acad. Sci. 681:165-167.

12. Beeson, D., et al. 1993. Primary structure of the muscle acetylcholine receptor: cDNA cloning of the $\gamma$ and $\varepsilon$ subunits. Eur. J. Biochem. 215:229-238.

13. Jacobson, L., Beeson, D., Tzartos, S., and Vincent, A. 1999. Monoclonal antibodies raised against human acetylcholine receptor bind to all five subunits of the fetal isoform. J. Neuroimmunol. 98:112-120.

14. Beeson, D., Amar, M., Bermudez, I., Vincent, A., and Newsom-Davis, J. 1996. Stable functional expression of the adult subtype of human muscle acetylcholine receptor following transfection of the human rhabdomyosarcoma cell line TE671 with cDNA encoding the $\varepsilon$ subunit. Neurosci. Lett. 207:57-60.

15. Hamill, O.P., Marty, A., Neher, E., Sakmann, B., and Sigworth, F.J. 1981. Improved patch-clamp techniques for high-resolution current recordings from cells and cell-free membrane patches. Pflugers Arch. 391:85-100.

16. Magleby, K.L., and Pallotta, B.S. 1983. Burst kinetics of single calciumactivated potassium channels in cultured rat muscle. J. Physiol. 344:605-623.

17. Ohno, K., et al. 2001. Choline acetyltransferase mutations cause myasthenic syndrome associated with episodic apnea in humans. Proc. Natl. Acad. Sci. USA. 98:2017-2022.

18. Ohno, K., et al. 1997. Congenital myasthenic syndromes due to heteroallelic nonsense/missense mutations in the acetylcholine receptor $\varepsilon$ subunit gene: identification and functional characterisation of six new mutations. Hum. Mol. Genet. 6:753-766.

19. Pedersen, S.E., and Cohen, J.B. 1990. $d$-Tubocurarine binding sites are located at $\alpha-\gamma$ and $\alpha-\delta$ subunit interfaces of the nicotinic acetylcholine receptor. Proc. Natl. Acad. Sci. USA. 87:2785-2789.

20. Chiara, D.C., and Cohen, J.B. 1992. Identification of amino acids contributing to high and low affinity $d$-tubocurarine sites on the Torpedo nicotinic acetylcholine receptor subunits. Biophys. J. 61:A106.

21. Prince, R.J., and Sine, S. 1996 . Molecular dissection of subunit interfaces in the acetylcholine receptor. J. Biol. Chem. 271:25770-25777.

22. Corringer, P.-J., et al. 1995. Identification of a new component of the agonist binding site of the nicotinic $\alpha 7$ homooligomeric receptor. $J$. Biol. Chem. 270:11749-11752.

23. Engel, A.G., Ohno, K., and Sine, S. 1999. Congenital myasthenic syndromes. In Myasthenia gravis and myasthenic disorders. A.G. Engel, editor. Oxford University Press. Oxford, United Kingdom. 251-297.

24. Beeson, D., and Newsom-Davis, J. 2000. Mutations affecting muscle nicotinic acetylcholine receptors and their role in congenital myasthenic syndromes. In Channelopathies: common mechanisms in aura, arrbythmia and alkalosis. F. Lehmann-Horn and K. Jurkat-Rott, editors. Elsevier Science B.V. Amsterdam, The Netherlands. 85-114.

25. Abicht, A., et al. 2000. Genetic analysis of the entire $\varepsilon$-subunit gene in 52 congenital myasthenic families. Acta Myologica. 19:23-27.

26. Sieb, J., et al. 2000. Severe congenital myasthenic syndrome due to homozygosity of the $1293 \mathrm{ins} G \varepsilon$-acetylcholine receptor subunit mutation. Ann. Neurol. 48:379-383.

27. Quiram, P., et al. 1999. Mutation causing congenital myasthenia reveals acetylcholine receptor $\beta / \delta$ subunit interaction essential for assembly. J. Clin. Invest. 104:1403-1410. 